\title{
Clinical Care for the Patient with Heart Failure: A Nursing Care Perspective
}

\section{Kristofina Amakali*}

Department of Health Science, University of Namibia, Windhoek, Namibia

\begin{abstract}
Providing clinical nursing care to a patient with a heart disease requires the application of the knowledge of disease process which informs the nursing intervention. This paper presents a discussion on some priority aspects of clinical care for the patient with heart failure from the perspective of nursing care as summarized in the figure below. A brief summative overview of the disease process and the central concept in heart failure as fundamental to the conceptualization of clinical care needs for a patient with heart failure was presented. Application of the framework of the nursing process in providing clinical nursing care for a patient with a heart disease and the application of ethical principles were discussed. Thus, competent and therefore safe clinical care for a patient with heart failure requires comprehensive nursing care which embraces both the scientific approach and ethics of care.
\end{abstract}

Keywords:Heart failure; Clinical; Nursing care

\section{Introduction}

The heart is a vital organ of the cardio-vascular system which is responsible for the maintenance of a balanced blood circulation throughout the body for the purpose of delivering oxygen and nutrients to the body systems, thereby ensuring the end-organ functioning and the elimination of the metabolic wastes from the body [1-5].

Before discussing the nursing care for a patient with heart failure, a brief summary of the overview of the disease process or the pathophysiology of heart failure is presented within the nursing perspective, an understanding of which informs a nursing care plan. Heart failure (right or left) is the inability of the heart to maintain a balanced circulation of the blood in the body $[2,3]$. The right sided heart failure is mainly characterized by the inability to maintain sufficient venous return, followed by systemic congestion, retention of metabolic wastes and the resultant edema and metabolic acidosis $[1,2,4]$. The left sided heart failure is characterized by a lowered cardiac output, followed by lowered delivery of oxygen and nutrients to the body systems. This result into lowered body metabolism, as may be characterized by lowered systemic output, such as low body temperature (hypothermia) among others. The inability of the left ventricle to empty itself causes pulmonary congestion and this result into poor gases exchange, low oxygen tension in the body-hypoxia as may be evidenced by visible cyanosis or by presentation of the signs of systemic hypoxia, such as general weakness $[5,6]$.

In summary, the core or central concept in right heart failure is that the patient is subjected to systemic venous congestion and retention of metabolic wastes which produce edema and metabolic acidosis. The central concept in left heart failure is that the patient is subjected to poor delivery of oxygen and nutrients to the body systems and pulmonary congestion, followed by low metabolism and hypoxia respectively.

Based on the disease process, the nurse should be able to conceptualize the clinical care needs for a patient with heart failure, design and implement a care plan with the interventions of which help to moderate the disease process and maintain homeostasis of the hemodynamics for the patient. Therefore, the clinical nurse should apply the perspectives of a nursing process to design a clinical care plan for a patient with heart diseases as discussed in the next sessions.

\section{Goals and Objectives}

The goal of this manuscript is to discuss the priority aspects for clinical nursing care for a patient with heart failure. The specific objectives for the manuscript were to conduct a literature review on the clinical nursing care for patients with heart failure and to describe the priority aspects for clinical nursing care for a patient with heart failure.

\section{Methods}

A systematic investigation of available textbooks was conducted in regard to the clinical nursing care for patients with heart diseases.

\section{Target population}

The target population for the concept "nursing care for a patient with heart disease" was all the available textbooks on medical nursing care which describe "heart failure" as an illness and discuss clinical management for patients with heart diseases.

\section{Sampling method and sample}

Selected textbooks on medical nursing care were consulted. A purposeful sampling method was used and textbooks on medical nursing care which discuss care for patients with heart failure were done. A total of thirteen (13) resources were utilized to reflect on the objectives of the study.

\section{Data analysis and findings}

The main themes on clinical care for patients with heart diseases that were identified from the sources were: monitoring of vital the signs for a patient with heart failure and the likely findings, life styles modification for the patient, diet modification for the patient,

*Corresponding author: Kristofina Amakali, School of Nursing and Public Health Faculty of Health Science, University of Namibia, Windhoek, Namibia, Tel: 00264 (61) 206 3297; Fax: 264-61-206 397; E-mail: kamakali@unam.na

Received March 23, 2015; Accepted: May 15, 2015; Published: May 20, 2015

Citation: Amakali K (2015) Clinical Care for the Patient with Heart Failure: A Nursing Care Perspective. Cardiol Pharmacol 4: 142. doi:10.4172/2329-6607.1000142

Copyright: (c) 2015 Amakali K. This is an open-access article distributed under the terms of the Creative Commons Attribution License, which permits unrestricted use, distribution, and reproduction in any medium, provided the original author and source are credited. 
administration of medications and oxygen therapy, coordination of the diagnostic examinations for efficient clinical management of the patient with heart failure, provision of counseling and health education to the patient and the family and the application of ethical principles in caring for the patient with heart failure.

\section{Discussion of the Findings}

The information under the themes on clinical care for a patient with heart failure imply that they can be organized under the phases of a nursing care process in order to facilitate as systematic care to a patient. Therefore, in the next sessions presented the discussions on the identified themes on clinical care for a patient with failure.

In the assessment phase, the nurse collects the subjective data from the patient and objective data about the actual and potential clinical problems for the patient, guided by the concept of "heart failure". The collected data are analyzed or interpreted and nursing diagnoses are formulated as the indicators for the patient's need for care. During planning phase, the nurse identifies the goals, objectives for care, the nursing interventions to realize the set goals and objectives and the outcomes of the caring interventions. The goals are conceptualized as to facilitate the care of the patient with a heart failure through multidisciplinary care interventions, to facilitate the patient's self-care and improve quality of life for the patient.

The nursing interventions are designed to meet the specific needs for caring as articulated in the nursing diagnosis and are implemented during the "implementation phase" of the nursing process, and their effectiveness in bringing about the desired outcomes would be evaluated during the "evaluation phase" of the nursing process. In that regard, nursing interventions for a clinical nursing care plan for a patient with heart failure should include among others, the priorities of: monitoring of vital signs, modification of patient's life styles, diet modification for the patient, administration of medications and oxygen therapy, the coordination of the diagnostic examinations and the provision of health education to the patient and the family. All these interventions should be implemented within the realm of the applicable ethical principles of caring $[4,6]$. The next sessions provide the detailed descriptions of the implementation of each of the stated priority aspects of clinical nursing care for a patient with heart failure.

\section{Theme 1: Monitoring of vital the signs for a patient with heart failure and the likely findings}

Low cardiac output which characterizes the left heart failure affects the vital signs adversely. Therefore, the nurse ought to monitor the vital signs, note the changes-likely subnormal, record, adjust the nursing care plan and report the abnormality to the physician. The status of the patient's vital signs as explained in the next sessions does inform the nurse interventions and the adjustment of treatment by the physician.

Pulse patterns: The pulse rate is likely to be high, as a compensatory mechanism to low cardiac output and hypoxia. The volume (pressure of the pulse) is likely to be shallow, of course due to low cardiac output. Furthermore, the patient presents with arrhythmia, indicating uncoordinated cardiac output by poor performing heart [1].

Blood pressure: The blood pressure is likely to be subnormal. While the systolic pressure is designated to be lower than normal, the diastolic pressure may be disproportionately elevated - thus signifying circulatory congestion due to poor venous return by the weakened heart.

Saturation: The patient's saturation may be lower than normal, i.e. less than $90 \%$, following low cardiac output with low oxygen distribution and pulmonary congestion in the left heart failure [1].

Respiration: The respiration may be fast to compensate for hypoxia, yet it is shallow because of lack of energy in the body [1].

Body temperature: As a result of low oxygen in the body, the patient's metabolism is lowered. Hence the patient's body temperature is likely to be subnormal [1]. The nurse should therefore encourage bed rest and provide in basic needs for the patient to avoid strenuous activities which increase the demands for oxygen in the body. Furthermore, the patient's body temperature should be conserved by providing warm clothes and warm beddings and oxygen therapy should be administered to relieve hypoxia and support the metabolism [3]. Additionally, when applicable, the nurse should institute independent nurse interventions to address the identified abnormal findings, or else she/he notifies the physician for necessary adjustment of the medical treatments.

\section{Theme 2: Life styles modification for the patient}

It is essential for the patient to modify his/her lifestyle, especially with regard to activities or behaviour and the emotions, all which may increase workload on the heart. The nurse should therefore implement both the provisional nursing interventions for the patient's life modification and advice the patient to modify his/her lifestyles for positive health outcomes. Provisionally, the nurse should create a caring environment which promotes rest for the patient in order to prevent the demand for oxygen, should the patient be overactive. Noise pollutions should be avoided to ensure emotional rest for the patient [3]. Equally, air pollution should be avoided to prevent respiratory distress, which can compound the eminent hypoxia. Therefore, the patient should be advised to avoid both active and passive smoking. Additionally, the patient should be advised to avoid consumption of alcohol because alcohol related toxins compounds to already retained metabolic wastes and as a result, increases the risk for metabolic acidosis [3].

\section{Theme 3: Diet modification for the patient}

Diet plays a significant role in maintenance of health for a patient with heart failure. The patient needs to modify his/her diet to comply with the golden rule of low salt, fat free formula. Therefore, while at the hospital, the patient should be provided with meals that are salt free and fat free. Salt free diet minimizes the risk of fluid retention which places a load on the ailing heart and fat free diet minimizes the risk for ischemic heart diseases. The food should be soft prepared in order to minimize the effort for mechanical digestion of hard foodstuff, which creates demand for energy, yet in a patient with a compromised body metabolism. Small servings of meals are recommended. Large meals place pressure on the heart, thus increases the workload. The patient should be advised to maintain a Semi-Fowler position after every meal in order to alleviate pressure on the heart. Caloric food should be provided to supplement the energy. Vitamin rich food should be offered to improve the patient's immunity because the patient's immunity is low owing to the low body metabolism [6].

\section{Theme 4: Administration of medications and oxygen therapy}

In a clinical setting, the medications for symptomatic treatment of heart failure are prescribed by the physician. The nurse's task is to administer the medications according to the prescription. However, the nurse is expected to have the knowledge of pharmacological namely, the therapeutic and side effects of such medications, in order to ensure progress of the patient and to prevent treatment related 
setbacks. If administered without discretions, the medications for treatment of heart failure can have lethal effects. The nurse would therefore be accountable for his his/her nursing actions with regard to the administration of prescribed medications.

The most common prescribed medications for the patients with heart disease are:

Digitalis, such as Digoxine: To improve contractions of the heart muscles and cardiac output $[1,4,8]$.

Diuretics, such as Furosemide: To enhance the elimination of excess fluid from the body, and by so doing, to prevent systemic edema such as pulmonary edema $[1,4,8]$.

In that regard, the nurse ought to evaluate the patient for the side effects related to each of the medications. In the next sessions, brief illustrations of nursing care implications to some of the medications for treatment of heart failure are provided.

Angiotensin converting enzyme inhibitors (ACE Inhibitor): To prevent the conversion of Angiotensin 1 to Angiotensin 2, thereby preventing reabsorption of salt and fluid by Aldosterone $[1,4,8]$.

Digoxine: is a digitalis which improves the effort of the heart contractions and the rhythms. It enables these therapeutic effects by slowing down the pace of the heart rate and as a result, stabilizes the heart rhythms. Thus digoxine toxicity can cause the patient to develop bradycardia and heart arrest as well. Therefore, the nurse should monitor the patient's pulse patterns to rule out bradycardia and arrhythmia before and after administering digoxine, and any abnormal findings should be promptly reported to the physician $[1,4,8]$.

Furosemide: is a diuretic which enhances the elimination of excess fluid from the body through urination and thereby also improves kidneys flush out. The desired therapeutic effect (elimination excess fluid) goes with the loss of essential electrolytes, especially sodium and potassium. Loss of sodium risks dehydration and hypovolemia, while the loss of potassium puts the patient at risk for arrhythmia, yet in the already ailing heart. Given the probable side effects of these medications, the nurse ought to assess the patient for the signs of dehydration, such as dry skin and mucosa and for low blood pressure and thread pulse. Similarly, the patient has to be monitored for the signs of imbalanced serum potassium, such as complains of palpitations, muscle cramps and evidences of arrhythmias $[1,4,8]$.

Angiotensin converting enzyme inhibitors (ACE Inhibitor): These types of medications prevent the conversion of Angiotensin 1 to Angiotensin 2 from the juxta glomeruro apparatus of the kidneys. As a result of a controlled or minimal angiotensin 2, there is less release of the aldosterone from the adrenal cortex of the kidneys. Less aldosterone implies reduced reabsorption of water and salt by the kidneys. The result is the loss of excess body fluid and minimization of the risk for systemic edema. Nevertheless the desired therapeutic effect, ACE Inhibitor can cause dehydration and electrolytes depletion of a magnitude. Therefore, like in the case of other diuretic, the nurse has to monitor the patient for the signs of imbalanced serum electrolytes $[1,4,7,8]$.

In addition to the medications, the patient is administered oxygen to ensure sufficient saturation. In that regard, care should be taken to avoid overflow of oxygen in order to prevent hyperinflation of the lungs and the risk for emphysema. The use of a nasal cannula for oxygen therapy ensures sufficient ventilation, while minimizing the sensations of suffocation that could be occasioned by the oxygen masks.

\section{Theme 5: Coordination of the diagnostic examinations for efficient clinical management of the patient with heart failure}

The justification and prescriptions of the diagnostic tests for a patient with a heart failure in a clinical setting is within the jurisdiction of the physician. The nurse's basic responsibility is to oversee that the diagnostic procedures are executed and the results are available for the interpretation by the physician in order for the physician to adjust the treatment accordingly. The most common diagnostic tests are the chest $\mathrm{x}$-rays, Electro-Cardio Graph (ECG) and the laboratory blood tests. The nurse's responsibilities towards these tests are explained in the next sessions [9].

Chest $\mathrm{x}$-ray and ECG: The chest $\mathrm{x}$-ray is ordered to rule out the enlargement of the heart and pulmonary congestion, especially when the patient suffers from left heart failure. Likewise, the ECG would detect the abnormalities of heart contractions as may be indicated by deviant ECG graphs. Therefore, on reception of such results, the nurse has to inform the physician on the availability of the results for punctual initiation or adjustment of medical treatment $[1,3,4]$.

Laboratory blood tests: Full Blood Count (FBC), Urea and Electrolytes (U\&E) are the most ordered blood tests for heart failure $[1,4]$. The purpose of Full Blood Count is among others, to verify the quantity of the red blood cells to ensure oxygen carrying capacity of the body to counteract hypoxia which is eminent in patient with heart failure. White blood cells are monitored to rule out low immunity and the risk for potential infection as a result of low metabolism that prevails in heart failure. The Urea is monitored to rule out possible retention of urea and other metabolic wastes owing to low venous return, a phenomenon which can cause metabolic acidosis. Electrolytes are monitored to rule out the imbalances of both the intracellular and extracellular electrolytes, the imbalances of which can risk arrhythmia and heart arrest since the heart is already in a failure status. Nevertheless, on reception of the blood results, the nurse should be able to interpret the results against the given laboratory references and identify the cases that need emergency attention of the physician [9].

\section{Theme 6: Provision of counseling and health education to the patient and the family}

Patients with heart failure should be counselled to accept and adapt to their health status. Acceptance helps the patient to make use of the potential he/she is left with, make a constructive adaptation and therefore to live positively with the illness [10]. Patients with heart disease should also be provided with health education to enable them maintaining self-care at home. Health education for a patient with heart failure should be organized around the issues of: self-monitoring, lifestyle modification, diet, medication self-administration and followup treatment, thus most of which are continuations of the aspects of clinical care in the hospital $[1,3,8,10,11]$.

As regard self-monitoring, the patient should be informed that heart failure causes general slow circulation, hypoxia and low systemic output. As a result, she/he may experiences the symptoms such as palpitations, dizziness, light-headedness, shortness of breath, weakness and coldness [5]. He/she therefore needs to observe development of oedema, difficulty breathing, while rest and keep him / herself warm. Intensification of the symptoms should be reported to the nearest health care facility [11]. The patient is advised to change any risky lifestyle such as alcohol consumption, tobacco smoking and avoid performance of strenuous activities, such as sport and emotional upsets. Most importantly, the diet for a patient with a heart failure should be discussed. In this regard, the local foodstuff that meets the requirement 
for a cardiac diet should be identified and the family members should be explained how to prepare the meal for the patient using foodstuffs that are available in the household. More emphasis should be placed on the restriction of salt to prevent oedema. The patient is advised to eat nutritious diet with high calorie in order to improve thrive and livelihood [7]. Dosages of medications and intervals of medication administration should be demonstrated and explained to the patient. The patient is encouraged to adhere to the treatment regiment, not to run out of medications and to maintain punctual follow up treatments [8-10]. Family members should be involved in all the facets of health education for the patient for them to provide support to the patient at home $[4,8,10,11]$. Recording of caring interventions provided to the patient is an essential integral part of clinical care.

\section{Theme 7: Applications of ethical principles in caring for the patient with heart failure}

Patients are human beings with personal values, beliefs and cultural principles. Therefore, a patient with heart failure as an individual should be provided professional care which is appropriate to his/her needs for care, ethically guided and relevant to his or her context. In that regard, the ethical principle of justice, autonomy and respect for a person, veracity, beneficence and the principle of no maleficence should be respected [12]. As regard justice, nursing care interventions ought to be planned and implemented in according the demands for care which are incurred by the illness [12].

The heart provides for the most basic need for life-the delivery of oxygen to the body systems to sustain the body metabolism. Hence, it is rationally acceptable to prioritize providing the care to a patient with heart failure in order to meet this basic and most vital need. However, as a human being and when applicable, the patient's right to selfdetermination should be respected. The patient's opinions regarding the treatment choices should be respected. Appropriate and honest information should be provided for the patient to make informed decision with regard to the treatment. However, the nurse should judge the extent to which truthiness should be applied in order to avoid an adverse situation that may arise if the patient is not ready to accept the truth about the illness or the treatment outcomes. Beneficence refers to the goodness that can be driven from the care interventions. In conjunction with truthiness, the need for adherence to treatment regimen should be discussed with the patient [13]. This in return helps the patient to take a role in his/her treatment and maintenance of his/ her own health. No maleficence refers to "no harm" to the patient. Harm to the patient in a clinical setting can be intentionally, such as the harm that are occasioned by nurses' negligence of their duty to care for the patient. On the other hand, unintentionally "harm" to the patient may be occasioned through the provision of specific nursing interventions to the patient. In both cases, the nurses should be vigilant to prevent any of the "harms" from happening. Intentionally, the nurse should design appropriate nursing interventions and prioritize the punctual implementation thereof. With regard to unintentional harm, nurses should avoid potential errors that may be occasioned through the administration of the medications, many of which can cause lethal side effects if not administered correctly $[12,13]$.

After the implementation of the caring interventions, the nurse conducts the evaluation of the impacts of the caring interventions on the patient. The efficiency and sufficiency of the implementation of the designed multidisciplinary caring interventions are appraised and the attainment of the set goals are evaluated as may desirable be evidenced by the patient's improved quality of life and the ability to self-care.

\section{Summary and Conclusion}

This paper presents a discussion on some priority aspects of clinical care for the patient with heart failure from the perspective of nursing care as summarized in the Figure 1 below. A brief summative overview of the disease process and the central concept in heart failure as fundamental to the conceptualization of clinical care needs for a patient with heart failure was presented. The findings were discussed as themes within the framework of the nursing process in providing clinical nursing care for a patient with a heart disease and the application of ethical principles were discussed.

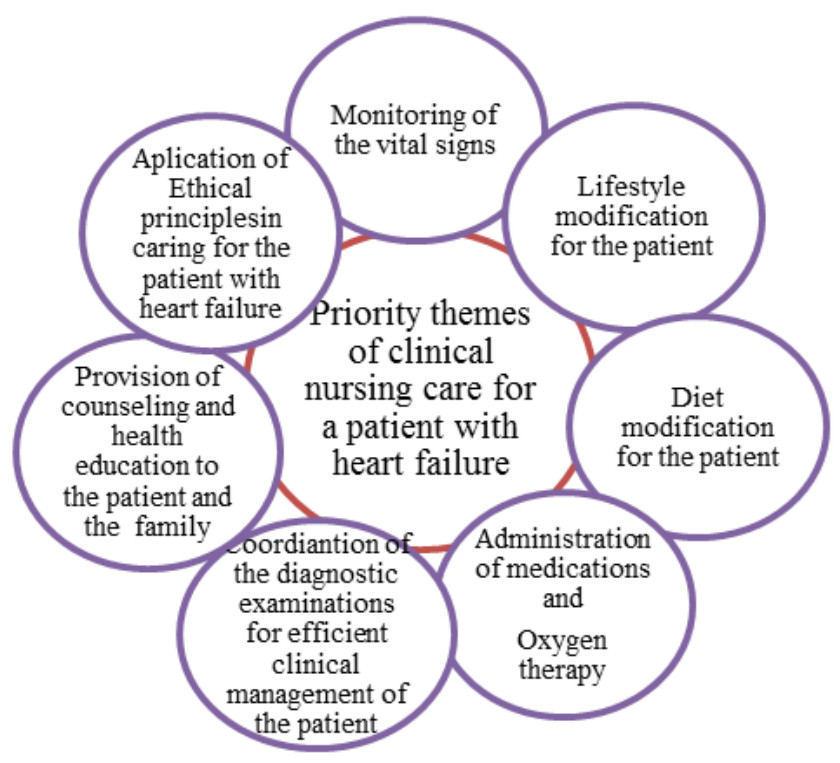

Figure 1: Priority themes for clinical nursing care for a patient with heart failure 
Citation: Amakali K (2015) Clinical Care for the Patient with Heart Failure: A Nursing Care Perspective. Cardiol Pharmacol 4: 142. doi:10.4172/23296607.1000142

Page 5 of 5

\section{References}

1. Smelter SC, Bare BG, Hinkle JL, Cheever KH (2010) Brunner \& Suddarth's Textbook of Medical-Surgical Nursing. (12thedn), Lippincott Williams \& Wilkins, Philadelphia

2. Kumar V, Ababas AK, Fausto N, Mitchelle RN (2007) Robbins Basic Pathology. International Edition. (8thedn), Saunders Elsevier, United States.

3. Mogotlane S, Shauke M, Matlakala M, Mokoena J, Young A (2013) Juta's Complete Textbook of Medical Surgical Nursing. (1stedn), Juta, Cape Town.

4. Thompson JM, McFarland GK, Hirsh JE, Tucker SM (2002) Mosby's Clinical Nursing. (5thedn), Mosby, Philadelphia.

5. Hurst M (2011) Medical-Surgical Nursing Review. (1stedn), McGrawHill, New York.

6. Stellenberg EL, Bruce JC (2007) Medical-Surgical Nursing for Hospital and Community. (African edn), Churchill Livingstone, South Africa.
7. Pretorius S, Sliwa K, Ruf V, Walker K, Stewart S (2012) Feeding the Emergence of Advanced Heart Diseases in Soweto: A Nutritional Survey of Black African Patients with Heart Failure. Cardiovascular Journal of Africa 23: 245-250.

8. Barber P, Robert D (2009) Essential of Pharmacology for Nurses. (2ndedn), McGrawHill, Open University press, Glasgow.

9. Malarkey LM, McMorrow ME (2005) Nursing Guide to Laboratory and Diagnostic Tests. (2ndedn), St. Louis: Elsevier Saunders, United States.

10. Paul S (2008) Hospital discharge education for patients with heart failure: what really works and what is the evidence? Crit Care Nurse 28: 66-82.

11. Strömberg A (2005) The crucial role of patient education in heart failure. Eur J Heart Fail 7: 363-369.

12. White $L$ (2001) Foundation of nursing: Caring for the whole person. (1stedn), Thompson Learning, Delmar.

13. Parahoo K (2006) Nursing Research: Principle, Process and Issues (6thedn) Palgrave MacMillan, New York. 